\title{
Inter-Orbital Effects in the Impure Pnictide Superconductors
}

\author{
A. Ciechan and K.I. Wysokiński* \\ Institute of Physics and Nanotechnology Centre, M. Curie-Skłodowska University \\ I. Radziszewskiego 10, PL-20-031 Lublin, Poland
}

\begin{abstract}
We study the local properties of the iron oxypnictides by means of the real space Bogolubov-de Gennes equations. Starting with the realistic energy spectrum and assuming small amount of impurities we calculate the energy dependence of the local density of states and spatial distribution of the local values of the superconducting energy gap. We pay particular attention to the role of the inter-orbital scattering of pairs and the effect of impurity scattering on the superconducting state. The effect of inter-orbital impurities depends on the relative signs of the order parameters related to two orbitals in question. For opposite signs impurities produce bound states inside the gap, while for the same sign of the order parameters they hardly affect the superconductor. The results obtained for impure systems have been shown as maps of the order parameters and local density of states. They well compare with the existing scanning tunnelling microscopy spectra of the iron pnictides.
\end{abstract}

PACS numbers: 71.10.Fd, 74.20.-z, 74.81.-g

\section{Introduction}

The multicomponent iron oxypnictides [1, 2] represent the new family of multiband superconductors with relatively high critical temperature. They are layered systems with superconducting carriers arising from $\mathrm{Fe}-\mathrm{As}$ planes. According to band structure calculations there exist two hole Fermi surfaces centred around $(0,0)$ and two electron Fermi surfaces around $(\pi, \pi)$. The minimal model of these systems has thus to feature at least two Fermi surfaces. The structure of the order parameter, its symmetry and the mechanism of superconductivity in these materials are still the matter of experimental and theoretical investigation. In particular, there are conflicting results concerning the superconducting gap. The proposals range from single-gap [3] to multi-gap structure [4-6], from nodal [5] to nodeless $[3,6]$ order parameter in reciprocal space.

The theoretical models of superconductivity in these materials are also inconsistent. Some of the results indicate the standard $s$-wave symmetry of the order parameter [7], but most of them suggest the existence of unconventional superconductivity with $s_{ \pm}$or $d$-wave symmetry [8-10]. However, the importance of few bands close to the Fermi energy and the leading role of inter-band interaction are fairly well established. It is interesting to note that similarly as in high temperature superconductors, the scanning tunnelling microscopy (STM) measurements reveal nanoscale inhomogeneities in iron oxypnictides. The superconducting gap magnitude varies by about $\pm 20 \%$ in the doped samples [11-14].

\footnotetext{
* corresponding author; e-mail: karol@tytan.umcs.lublin.pl
}

The response of the clean system to impurities gives an important information useful to discriminate between the types of order parameter [15-17]. However, even the best samples of iron pnictides studied so far are rather dirty. The presence of various types of impurities and other types of disorder strongly affects their properties and makes the studies very timely and important. To discuss some of the issues we use the many-orbital model of the iron oxypnictide and calculate the local properties of the system. The model we are using is primarily applicable to the 1111 family of electron doped pnictide superconductors: $\mathrm{LnFeAsO}_{1-x} \mathrm{~F}_{x}$, where $\mathrm{Ln}=\mathrm{La}, \mathrm{Ce}$, $\mathrm{Pr}, \mathrm{Nd}, \mathrm{Sm} \ldots$

The organisation of the rest of the paper is as follows. Section 2 presents the Hamiltonian of the two orbital tight-binding model and the Bogolubov-de Gennes $(\mathrm{BdG})$ equations used to solve it. The changes induced in the superconductor by the single inter-orbital impurity or the finite concentration of such impurities are considered in Sect. 3. We present our results as maps of the local order parameters and the local densities of states. Section 4 contains the conclusions and the discussion of the results in relation to the STM measurement for Fe-based superconductors.

\section{Two-orbital model for superconductors}

We study the inhomogeneous two-orbital model with few hopping parameters $t_{i, j}^{\lambda, \lambda^{\prime}}$ and the two main types of local pairing interactions of negative Hubbard $U$ variety, which we call intra- $\left(U_{i}^{11}, U_{i}^{22}\right)$ and inter-orbital $\left(U_{i}^{12}\right)$. The symbols $\lambda, \lambda^{\prime}=1,2$ refer to two orbitals and $i, j$ denote sites in the cluster. To model impurity scattering we introduce the on-site potential in the form 
$\sim V_{\mathrm{imp}}^{\lambda \lambda^{\prime}} c_{i \lambda \sigma}^{+} c_{i \lambda^{\prime} \sigma}$, which may be of intra-orbital $\left(\lambda=\lambda^{\prime}\right)$ or inter-orbital $\left(\lambda \neq \lambda^{\prime}\right)$ type. The long range impurities are modelled by allowing for non-zero value of $V_{\text {imp }}$ at the location of impurity and neighbouring sites.

We model the energy spectrum near the Fermi level of oxypnictide superconductors [18] by assuming that $d_{x z}$ and $d_{y z}$ (which we denote by the symbol $\lambda=1,2$ ) orbitals of Fe ions play the most important role. We take into account the nearest neighbour hoppings $t_{i, i \pm x}^{11}=$ $t_{i, i \pm y}^{22}=-t$ and $t_{i, i \pm y}^{11}=t_{i, i \pm x}^{22}=1.3 t$ between the same orbitals and the next nearest neighbour hoppings $t_{i, i \pm x \pm y}^{11}=t_{i, i \pm x \pm y}^{22}=-0.85$. Hybridization between two orbitals has a form $t_{i, i \pm(x+y)}^{12}=-t_{i, i \pm(x-y)}^{12}=0.85 t$.

The model well describes electron and hole Fermi pockets on the large BZ [18] of the homogeneous system as well as the densities of states ratio $N_{\mathrm{h}} / N_{\mathrm{e}} \approx 2.6$ near the Fermi level. For nonzero but also for vanishing intra-band pairing interaction $U^{\lambda \lambda}$ one gets two order parameters, which in homogeneous case may have different phases $\Delta=\Delta_{1}= \pm \Delta_{2}$ on the two Fermi surfaces depending on the sign of the inter-band pair scattering term $\mp U^{12}[9]$.

To treat non-homogeneous system with two orbitals per site the Hamiltonian is best formulated in the real space as

$$
\begin{aligned}
H & =\sum_{i j, \lambda \lambda^{\prime}, \sigma}\left(-t_{i, j}^{\lambda \lambda^{\prime}}-\mu \delta_{i j} \delta_{\lambda \lambda^{\prime}}+V_{\mathrm{imp}}^{\lambda \lambda^{\prime}}\left(\boldsymbol{r}_{i}\right) \delta_{i j}\right) c_{i \lambda \sigma}^{+} c_{j \lambda^{\prime} \sigma} \\
& +\sum_{i, \lambda \lambda^{\prime}} U_{i}^{\lambda \lambda^{\prime}} c_{i \lambda \uparrow}^{+} c_{i \lambda \downarrow}^{+} c_{i \lambda^{\prime} \downarrow} c_{i \lambda^{\prime} \uparrow} .
\end{aligned}
$$

Here $c_{i \lambda \sigma}^{+}, c_{i \lambda \sigma}$ denote creation and annihilation operators of electrons with spin $\sigma=\uparrow, \downarrow$ at the lattice site $\boldsymbol{r}_{i}=i$ in the orbital $\lambda . \mu$ is the chemical potential, $t_{i, j}^{\lambda \lambda^{\prime}}$ are the hopping integrals between the same (if $\lambda=\lambda^{\prime}$ ) or different orbitals (if $\lambda \neq \lambda^{\prime}$ ). $U_{i}^{\lambda \lambda^{\prime}}$ are on-site interactions, which are attractive for $U_{i}^{\lambda \lambda^{\prime}}<0$. The dependence of the parameters on the position $\boldsymbol{r}_{i}$ allows one the examination of inhomogeneous systems.

The standard mean-field decoupling together with Bogolubov-Valatin canonical transformation lead to the BdG [17] for amplitudes $u_{\lambda \nu}\left(\boldsymbol{r}_{i}\right), v_{\lambda \nu}\left(\boldsymbol{r}_{i}\right)$ and eigenenergies $E_{\nu}$, which in turn allow the calculations of all observables [19] like local values of the order parameter and the density of states. For a two-orbital model the typical size of the cluster, which quite well reproduces [17] the properties of the bulk systems, is about $19 \times 15$.

\section{Inhomogeneous superconductors}

In this section we study the effects of nonmagnetic impurities randomly located in a small superconducting cluster of size $L=19 \times 15$ on the local properties of the system, starting with the simple case of a single impurity in the center of the cluster. We assume the mean number of particles in disordered system to be constant and equal to $n=2.15$. In this paper we consider only inter-orbital impurities and two different superconducting scenarios. Inter-orbital impurity means such one which scatters an electron from orbital 1 to 2 located at the same site $\boldsymbol{r}_{i}$ and vice versa. We show the maps of the local order parameters and the densities of states for two different situations: (a) $U^{11}=U^{22}=-|U|$ and $U^{12}=-|U|$ and (b) $U^{11}=U^{22}=-|U|$ and $U^{12}=|U|$. Two cases differ by the sign of inter-orbital pair scattering. In the clean system the pairing potential $|U|=0.8 t$ produces the gap $|\Delta| \approx 0.12 t$ in case (a), i.e. for attractive inter-orbital interaction. Roughly the same value of the gap in case (b) is obtained for $|U|=1.05 t$.

Figures 1a,c shows the maps of the normalized order parameters $\Delta / \Delta_{0}$ around inter-orbital impurity located at the centre of the cluster studied. Here $\Delta_{0}$ is the value in the clean system. The parts ( $b$ and $d$ ) of the figure show the local densities of states for the clean system (thick solid line), at the impurity site $(0,0)$ and neighbouring site $(1,1)$.
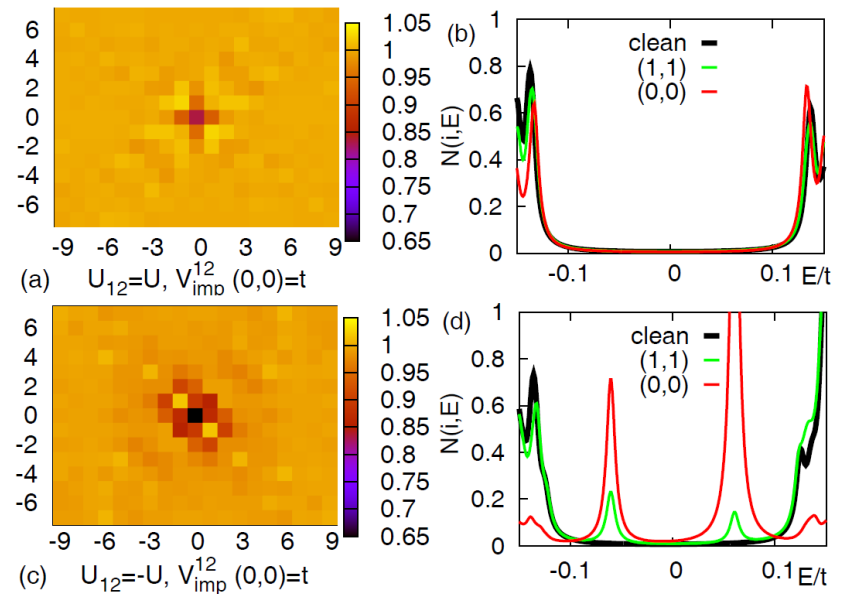

Fig. 1. The relative changes of the order parameters $\Delta / \Delta_{0}$ and the local densities of states for the case of the single inter-orbital impurity $V_{\mathrm{imp}}^{12}=t$ located at the site $\boldsymbol{r}=(0,0)$. Parts (a) and (b) refer to inter-orbital attractive interaction $U^{12}=-0.8 t$; parts (c) and (d) to repulsive $U^{12}=1.05 t$.

The changes of the order parameters $\Delta / \Delta_{0}$ at and around the impurity strongly depend on the sign of the inter-orbital interaction $U^{12}$. For repulsive $U^{12}$ the superconducting local gaps are more severely influenced by the impurity scattering than for attractive $U^{12}$. This is so because of different signs of the order parameters related to two orbitals for repulsive $U^{12}$. In this case there appear bound states inside the gap. Similar localized states have also been observed [20-22] in the presence of non-magnetic intra-orbital impurity. Here, the effect is much stronger, the bound states are located well inside the gap and are symmetrical around zero energy. Also interesting patterns in the changes of the order parameter are observed (Fig. 1). They reflect the symmetry of the hopping integrals between two $d_{x y}-d_{y z}$ orbitals.

To illustrate the properties of the two-orbital inhomogeneous superconductor we consider the sample with $15 \%$ randomly distributed impurities. Impurities extend over 


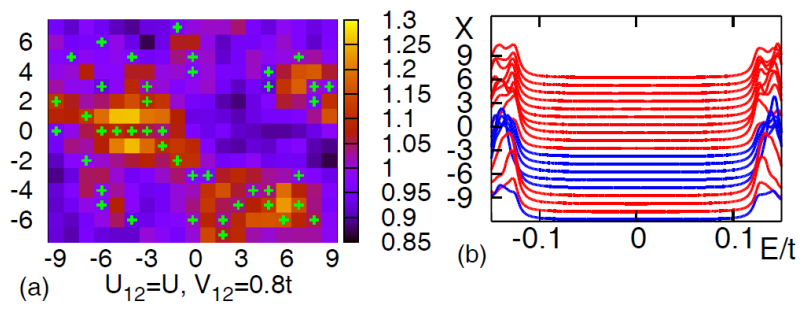

Fig. 2. The map of $\Delta / \Delta_{0}$ (a) and the local density of states along the line $y=0$ (b) for inter-orbital scattering $V^{12}=0.8 t$ and attractive pairing potential $U^{12}=-0.8 t$.

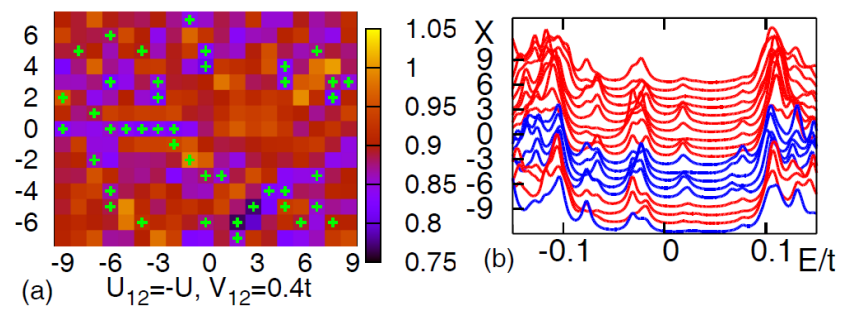

Fig. 3. The map of $\Delta / \Delta_{0}$ (a) and the local densities of states $N(x, y=0, E)$ (b) for inter-orbital scattering $V^{12}=0.4 t$ and repulsive $U^{12}=1.05 t$.

two lattice distances around the central one and their distribution is kept constant. Figure 2 shows the map of the relative order parameter $\Delta(\boldsymbol{r}) / \Delta_{0}$ (with $\Delta_{0}$ referring to the clean system) and the local densities of states along the line $y=0$ for attractive inter-band interaction $U^{12}=-0.8 t$ and impurity strength $V^{12}=0.8 t$, while Fig. 3 shows similar data obtained for repulsive $U^{12}=1.05 t$ and weaker impurities $V^{12}=0.4 t$. For $U^{12}<0$ the impurities affect the superconducting state relatively weakly. The decrease of $\Delta / \Delta_{0}$ at and its increase near the impurities is a combined effect of changes in the (local) density of states and the deformation of the superconducting wave function trying to preserve the condensation energy despite the impurity (cf. also Fig. 1a). However, one notices the appearance of the separate regions with high and low values of the order parameter. For $U^{12}>0$ even weak potential scattering leads to the in-gap bound states and suppression of superconductivity. The distribution of the gap values is more uniform in this scenario.

\section{Discussion and conclusions}

We have studied the effect of inter-orbital impurity scattering on the superconducting properties of iron pnictides described by the two-orbital model and intra-orbital or inter-orbital pairing interactions. The attractive inter-orbital pairing interaction leads to $s$-wave symmetry states $\Delta_{\lambda}$ with the same phases for $\lambda=1$ and $\lambda=2$, while the repulsive one to $s_{ \pm}$-state, which is characterised by the order parameters with different phases
$\Delta_{1}(\boldsymbol{r})=-\Delta_{2}(\boldsymbol{r})$. The impurities strongly affect the local properties of the material with $s_{ \pm}$-superconducting state. The bound states appear inside the gap and the average value of the order parameter strongly decreases with the strength of the impurity potential $V^{12}$, while the state with the same phase of the order parameter seems to be more robust against both intra-orbital and inter-orbital impurities [17].

The iron oxypnictide samples are certainly strongly disordered systems. Despite of that the local gaps measured by STM [11-14] do not change very drastically. This makes the inter-orbital pairing with repulsive $U^{12}$ and thus $s_{ \pm}$-superconducting state [9] rather unlikely. Our results favour the scenario with attractive inter-orbital pairing and $s$-wave state, which is protected against non-magnetic impurities by the Anderson-like mechanism.

\section{Acknowledgments}

This work has been partially supported by the Polish Ministry of Science and Education under the grant No. N N202 169836.

\section{References}

[1] Y. Kamihara, H. Hiramatsu, M. Hirano, R. Kawamura, H. Yanagi, T. Kamiya, H. Hosono, J. Am. Chem. Soc. 128, 10012 (2006).

[2] Y. Kamihara, T. Watanabe, M. Hirano, H. Hosono, J. Am. Chem. Soc. 130, 3296 (2008).

[3] T.Y. Chen, Z. Tesanovic, R.H. Liu, X.H. Chen, C.L. Chien, Nature 435, 1224 (2008).

[4] Y.-L. Wang, L. Shan, L. Fang, P. Cheng, C. Ren, H.-H. Wen, Supercond. Sci. Technol. 22, 015018 (2009).

[5] K. Matano, Z.A. Ren, X.L. Dong, L.L. Sun, Z.X. Zhao, G.-Q. Zheng, Europhys. Lett. 83, 57001 (2008).

[6] H. Ding, P. Richard, K. Nakayama, T. Sugawara, T. Arakane, Y. Sekiba, A. Takayama, S. Souma, T. Sato, T. Takahashi, Z. Wang, X. Dai, Z. Fang, G.F. Chen, J.L. Luo, N.L. Wang, Europhys. Lett. 83, 47001 (2008).

[7] O.V. Dolgov, I.I. Mazin, D. Parker, A.A. Golubov, Phys. Rev. B 79, 060502(R) (2009).

[8] K. Ishida, Y. Nakai, H. Hosono, J. Phys. Soc. Japan 78, 062001 (2009)

[9] I.I. Mazin, J. Schmalian, Physica C: Superconductivity 469, 9 (2009).

[10] Y. Bang, H.-Y. Choi, Phys. Rev. B 78, 134523 (2008).

[11] O. Millo, I. Asulin, O. Yuli, I. Felner, Z.-A. Ren, X.L. Shen, G.-C. Che, Z.-X. Zhao, Phys. Rev. B 78, 092505 (2008).

[12] Y. Yin, M. Zech, T.L. Williams, X.F. Wang, G. Wu, X.H. Chen, J.E. Hoffman, Phys. Rev. Lett. 102 , 097002 (2009).

[13] Y. Yin, M. Zech, T.L. Williams, J.E. Hoffman, Physica C: Superconductivity 469, 535 (2009). 
[14] F. Massee, Y. Huang, R. Huisman, S. de Jong, J.B. Goedkoop, M.S. Golden, Phys. Rev. B $\mathbf{7 9}$, 220517(R) (2009).

[15] A.A. Golubov, I.I. Mazin, Phys. Rev. B 55, 15146 (1997).

[16] V.G. Kogan, C. Martin, R. Prozorov, Phys. Rev. B 80, 014507 (2009).

[17] A. Ciechan, K.I. Wysokiński, Phys. Rev. B 80, 224523 (2009).

[18] S. Raghu, Xiao-Liang Qi, Chao-Xing Liu, D.J. Scalapino, Shou-Cheng Zhang, Phys. Rev. B 77, 220503 (2008).
[19] J B. Ketterson, S.N. Song, Superconductivity, Cambridge University Press, Cambridge 1999.

[20] W.-F. Tsai, Y.-Y. Zhang, C. Fang, J. Hu, Phys. Rev. B 80, 064513 (2009).

[21] D. Zhang, Phys. Rev. Lett. bf 103, 186402 (2009).

[22] T. Zhou, Xiang Hu, Jian-Xin Zhu, C.S. Ting, preprint available at arXiv:0904.4273v6. 